\section{Atypical presentation of thrombosis of a permanent pacemaker lead}

\author{
DRAŽEN ZEKANOVIĆ • VEDRAN CAREVIĆ • ZORISLAV ŠUŠAK • \\ GRGO GUNJAČA • ZORA SUŠILOVIĆ-GRABOVAC • IVO BOŽIĆ • \\ DAMIR FABIJANIĆ
}

DAMIR FABIJANIĆ $(\bowtie) \bullet$ VEDRAN CAREVIĆ • ZORA SUŠILOVIĆ-GRABOVAC • IVO BOŽIĆ

Split University Hospital Division of Cardiology Spinčićeva 1, 21000 Split, Croatia Phone: +38521556 766 Fax: + 38521556031 E-mail: damir.fabijanic@st.t-com.hr

DRAŽEN ZEKANOVIĆ • ZORISLAV ŠUŠAK

Division of Cardiology

General Hospital Zadar

GRGO GUNJAČA

Department of Pharmacology University of Split School of Medicine

\begin{abstract}
Pacemaker related infective endocarditis (PMIE) and pacemaker lead thrombosis (PMLT) are infrequent but potentially lethal complications of pacemaker (PM) therapy. Differences in clinical presentation, echocardiographic appearance and laboratory findings are usually helpful in making a confident diagnosis. On the other hand, atypical clinical and echocardiographic findings may complicate their differentiation and result in a therapeutic dilemma. We present a 70-year-old man with a permanent PM hospitalized because of a 7-day history of fever and weakness. Elevated inflammatory parameters and atypical echocardiographic findings resulted in a diagnostic dilemma between PMIE and PMLT. In this paper, we discuss the pathogenesis of these entities, their clinical presentation and therapy.
\end{abstract}

Key words: echocardiography, endocarditis, pacemaker, lead, thrombosis

\section{Introduction}

Pacemaker related infective endocarditis (PMIE) and pacemaker lead thrombosis (PMLT) are infrequent but potentially lethal complications of pacemaker (PM) therapy. $(1,2)$ PMIE often presents with fever, weakness, and elevated inflammation markers, $(1,3,4)$ while PMLT is usually an accidental echocardiographic finding, only occasionally presenting with right sided heart failure, rhythm disorder or pulmonary artery embolism. $(2,5,6)$ Differences in clinical presentation, in addition to characteristic echocardiographic appearances and laboratory findings, are usually helpful in distinguishing the aforementioned entities with confidence. However, atypical clinical and echocardiographic findings may sometimes complicate their differenti- ation and result in a therapeutic dilemma. Namely, in the majority of patients, PMIE must be treated with prolonged antibiotic therapy (4-6 weeks in most cases) and removal of the pacemaker. $(1,2,4)$ On the other hand, the treatment of PMLT only requires anticoagulant therapy. $(2,5)$

\section{Case report}

A 70-year-old man, who had a one chamber PM implanted 20 years ago, was hospitalized because of a 7-day history of fever and weakness. Eight years earlier, when the PM battery was exhausted, a new device was inserted. Because of incompatibility between the new device and the existing electrode, a new electrode was installed alongside the old one. Six weeks before this hospitalization the patient underwent transurethral prostatectomy with no antibiotic prophylaxis.
Physical examination and chest $\mathrm{X}$-ray revealed no abnormalities. Electrocardiography (ECG) revealed normal function of the PM in the VVIR mode. Inflammatory markers were elevated, including erythrocyte sedimentation rate $(50 \mathrm{~mm} / \mathrm{h})$, white blood cells $(15.2 \mathrm{x}$ $\left.10^{9} / \mathrm{L}\right)$ and C-reactive protein (65 mg/L). Other laboratory parameters were in the normal range.

On transthoracic (TTE) and transoesophageal echocardiography (TEE) two PM leads were demonstrated in the right cavities. A highly mobile, echodense, irregularly shaped mass sized 28x7 $\mathrm{mm}$ was attached to one of them with a narrow radix, while the more voluminous part was freely fluttering in the right atrium (figures $1 \mathrm{~A}$ and $\mathrm{B}$ ). There were no other echocardiographic abnormalities. Differential diagnoses of PMIE and PMLT were considered.

Blood cultures were negative. A urinary 
Figure 1. Echocardiographic findings, transthoracic (A) and transoesophageal (B). An echodense, irregularly shaped mass (thick arrow), sized $28 \times 7 \mathrm{~mm}$ was attached to one of pacemaker lead (thin arrow) with a narrow radius, while the more voluminous part was freely fluttering in the right atrium.
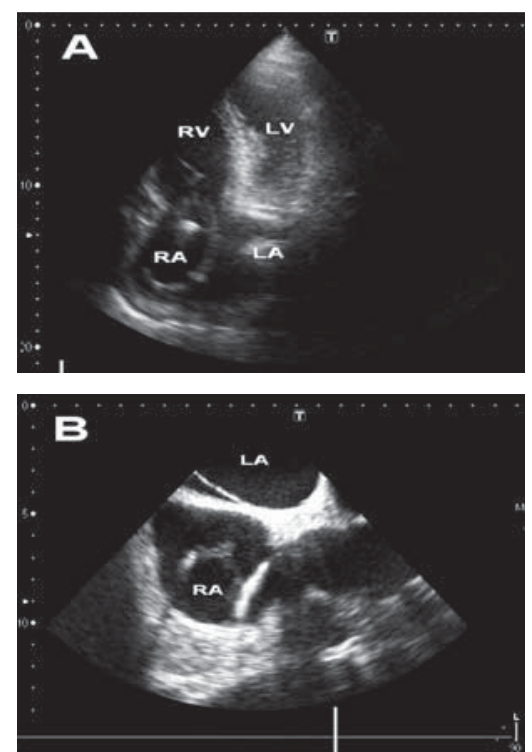

$L A$, left atrium; LV, left ventricle; $R A$, right atrium; RV, right ventricle.

infection, caused by Escherichia coli, was discovered. A three week course of anticoagulant therapy, with enoxaparin and warfarin, resulted in complete regression of the PM lead mass. The urinary infection resolved with co-trimoxazol. However, in order to prevent re-thrombosis, permanent anticoagulation with warfarin was recommended.

\section{Discussion}

Although PMIE or PMLT are relatively rare entities, the rising number of patients with implanted cardiac devices explains the increasing frequency of these potentially lethal complications of PM therapy. The rate of infection after implantation of permanent endocardial leads is estimated between $1 \%$ and $2 \%$. (1) At the same time, serious thrombotic and embolic complications are reported to occur in $0.6 \%$ to $3.5 \%$ of patients with permanent transvenous pacing leads. (2)

The PM lead alternates between an alien body tissue reaction, inflammation and fibrosis along the electrode, which are the basic mechanisms in the development of thrombi and endocardial vegetations. $(2,3)$ Also, risk factors for PMIE and PMLT include almost the same chronic conditions such as diabetes, malignancies, immunosuppressive therapy, and local factors related to the pacing system such as erosion of the PM pouch, and the number of previously inserted leads. $(5,7)$ Therefore, these clinical entities have some common pathophysiological mechanisms. Distinction between these two entities is based on typical clinical, laboratory and echocardiographic findings. $(5,8)$ PMIE must be suspected in the presence of unexplained fever in patients with a permanent PM, especially in patients who underwent a procedure accompanied by bacteremia (for example, transurethral prostatectomy, as was the case in our patient). $(4,9,10)$ As in other forms of infective endocarditis (IE), echocardiography and blood cultures are the cornerstone of PMIE diagnosis. $(4,9,10)$

On echocardiography, vegetations are often presented as circumscribed masses or clumps of echoes that arose from leaflet tips or electrode leads, either as an irregular area of highly reflective leaflet thickening or as more discrete, pedunculated mobile masses in the setting of electrode lead infection confirmed by imaging in more than one echocardiographic plane. (8) These characteristics may be helpful in the case of uncommon culture-negative IE in which the diagnosis of IE may be suggested on the basis of echocardiography even in the absence of, or certainly prior to, positive blood culture. $(8,10)$ Although TTE may identify lead vegetations, it has not been established as major diagnostic criteria for PMIE due to its low sensitivity. $(8,10)$ TEE is more helpful, it allows the exploration of the entire pacing system from the superior vena cava to the right ventricle. $(8,10)$ Also, TEE might be helpful in detecting other intracavitary masses (e.g. cardiac myxoma, mural thrombus). $(11,12)$

Blood cultures are positive in $77 \%$ of cases of PMIE. $(3,4,7)$ Staphylococci are the most frequent pathogens. $(7,9)$ It is important to note that Duke criteria are difficult to apply in these patients because of lower sensitivity. (10) Because septic pulmonary embolism is a very frequent complication of $\mathrm{PMIE}$, modifications of Duke criteria have been proposed. (10) Namely, according to modified Duke criteria, local signs of infection and pulmonary embolism are major diagnostic criteria for diagnosing PMIE. (10) The main mechanism of PMIE is contamination by local bacteriological flora at the time of device implantation. $(10,13)$ Then, the infection can spread along the electrode to the endocardium and the electrode tip. $(10,13)$ Hematogenous colonization of the PM conducting system during the course of a bacteremia from a distant focus has rarely been demonstrated since the pacing system is rapidly covered by neoendothelium and fibrous tissue, which prevents the foreign surface from being coated by microorganisms. $(7,9,10)$ However, in bacteraemic patients with fever and positive blood culture results, hematogeneous seeding of the device was presumed to be the mode of colonization of the system. $(7,9,10)$ The potential sources include infections at other sites, including the urinary, hepatobiliary, gastrointestinal, and respiratory tracts. $(7,10)$ However, it is important that new guidelines for the preventionand treatment of infective endocarditis do not recommend antibiotic prophylaxis in patients with a permanent pacemaker. $(10,14)$

Uslan et al. reported bacteremia in 75 of the 1524 patients with permanent PM in the full 30-year cohort. (7) The most common organisms causing bacteremia were Staphylococcus aureus (28\% of cases) and Escherichia coli (22\% of cases). (7) However, that study supports the consideration of device infection among patients with gram-positive bacteremia, especially bacteremia with S. aureus. (7) On the contrary, device infection, or the necessity for device removal, does not seem to be commonly found in patients with bacteremia caused by gram-negative bacilli. $(7,15,16)$ It is possible that differenti- 
al rates of infection during bacteremia caused by gram-positive and gramnegative microorganisms are caused by intrinsic pathogen-related factors (such as adherence factors and biofilm formation), device-specific characteristics, or host factors such as time to bacteremia after implantation, age or sex. (7) An alternative explanation is that device infection does occur with bacteremia due to gram-negative baciIli, but antimicrobial therapy is curative, even if the system is infected and not removed. (7)

Right atrial PMLT has been less frequently described, presenting either as an incidental echocardiographic finding or by symptoms of right-sided heart failure, obstruction or embolization of pulmonary artery. $(2,5,6)$ On echocardiography PMLT is occasionally presenting as immobile solid mass around the PM lead. $(2,5)$

Several mechanisms have been postulated in the pathogenesis of PMLT. The long-term residence of permanent PM leads may act as a continuous nidus for thrombus formation. $(2,5)$ Multiple PM wires (in cases of dual-chamber pacing or in the case in which a new electrode was installed alongside the old one, as in our patient) increase the total surface area of foreign material and risk for thrombus formation. (2) PM leads may produce a foreign-body-type reaction with subsequent inflammation and fibrosis along the course of the wire, and this may lead to thrombus propagation along the pacing lead. $(2,5)$ Furthermore, congestive heart failure, hypercoagulable states such as antithrombin, protein $\mathrm{C}$ and $\mathrm{S}$ deficiencies, thrombocytosis, malignancies, and possibly PM lead material itself, may predispose to thrombosis. (2) It has been shown that PM leads with different types of insulation have various thrombogenicity. Thrombogenicity of polyurethane leads may be lower than that of silicone leads. $(2,5)$ In our case, the thrombus was most likely a consequence of surface damage of the leads, caused by their mutual friction. The hemodynamic significance of the intracardiac clot depends on its size, ranging from one to several centimeters in diameter, and location. $(2,5,6)$ Atrial arrhythmias can occur if the sinoatrial area is involved. Similar to atrial myxomas, an intracardiac thrombus may cause functional tricuspid stenosis or insufficiency, and should be considered when refractory right ventricular failure is present. $(2,5,6)$

\section{Conclusion}

In the case of a PM lead mass in a febrile patient, clinical and echocardiographic suspicion should be directed towards thrombosis, as well as IE. After PMIE exclusion, for which negative blood cultures are the most important criteria, administration of anticoagulant therapy and gradual thrombus disappearance should solve the dilemma. Also, our case suggests that if, during the replacement of a PM, the lead cannot be substituted, permanent anticoagulant therapy is recommended in order to prevent PMLT, even in patients without associated thrombogenic risks.

\section{REFERENCES}

1. Cabell CH, Heidenreich PA, Chu VH, Moore CM, Stryjewski ME, et al. Increasing rates of cardiac device infections among Medicare beneficiaries: 1990-1999. Am Heart J 2004;147:582-6.

2. Barakat K, Robinson NM, Spurrell RA. Transvenous pacing lead-induced thrombosis: a series of cases with a review of the literature. Cardiology 2000;93:142-8.

3. Duval X, Selton-Suty C, Alla F, Salvador-Mazenq M, Bernard Y, Weber M, et al. Endocarditis in patients with a permanent pacemaker: a 1-year epidemiological survey on infective endocarditis due to valvular and/or pacemaker infection. Clin Infect Dis 2004:39:68-74.

4. Cacoub P, Leprince P, Nataf P, Hausfater P, Dorent R, Wechesler B, et al. Pacemaker infective endocarditis. Am J Cardiol 1998;82:480-4

5. Fabijanić D, Giunio L, Vujičić M, Vuković I, Ermacora R, Knežević N. Right atrial pacemaker lead thrombosis incidentally detected by transesophageal echocardiography. Coll Antropol 2005;29:159-61.

6. Wierzbowska K, Krzemińska-Pakuła M, Marszal-Marciniak M, Drozdz J, Zasłonka J, Kasprzak JD. Symptomatic atrial pacemaker lead thrombosis: detection by echocardiography and successful surgical treatment. Pacing Clin Electrophysiol 2001;24:391-3.

7. Uslan DZ, Sohail MR, St. Sauver JL, Friedman PA, Hayes DL, Stoner SM, et al. Permanent pacemaker and implantable cardioverter defibrillator infection. A population-based study. Arch Intern Med 2007;167:669-75.

8. SanFilippo AJ, Picard MH, Newell JB, Rosas E, Davidoff R, Thomas JD, et al. Echocardiographic assessment of patients with infective endocarditis. J Am Coll Cardiol 1991;18:1191-9.

9. Chamis AL, Peterson GE, Cabell CH, Corey GR, Sorrentino RA, Greenfield RA, et al. Staphylococcus aureus bacteriemia in patients with permanent pacemakers or implantable cardioverter-defibrillators. Circulation 2001:104:1029-33.

10. Habib G, Hoen B, Tornos P, Thuny F, Prendergast B, Vilacosta I, et al. Guidelines on the prevention, diagnosis, and treatment of infective endocarditis (new version 2009): the Task Force on the Prevention, Diagnosis, and Treatment of Infective Endocarditis of the European Society of Cardiology; European Society of Clinical Microbiology and Infectious Diseases; International Society of Chemotherapy for Infection and Cancer. Eur Heart J 2009;30:2369-413.

11. Fabijanić D, Rudež I, Kardum D, Radić M, Glavaš D, Lozo P. Pulmonary embolism due to the right atrial myxoma. Coll Antropol 2006;30:315-9.

12. Fabijanić D, Rudež I, Radić M, Unić D, Barić D, Kardum D. Pulmonary embolism due to the right atrial thrombus mymicking atrial myxoma. Chin Med J 2010;123:2843-5.

13. Da Costa A, Lelievre H, Kirkorian G, Celard M, Chevalier P, Vandenesch F, et al. Role of the axillary flora in pacemaker infections: a prospective study. Circulation 1998;97:1791-5.

14. Fabijanić D. Antibiotic prophylaxis of infective endocarditis in patients with moderate risk - rationale for and against Šin Croatianć. Lijec Vjesn 2010;132:62-4.

15. Bracke FA, Meijer A, van Gelder LM. Pacemaker lead complications: When is extraction appropriate and what can we learn from published data? Heart 2001:85:254-9.

16. del Rio A, Anguera I, Miro J, Mont L, Fowler VG Jr, Azqueta M, et al. Surgical treatment of pacemaker and defibrillator lead endocarditis. The impact of electrode lead extraction on outcome. Chest 2003;124:1451-9. 